\title{
Next-generation melanoma prevention efforts for overlooked populations and populations with health disparities: a South African perspective
}

${ }^{1}$ Environment \& Health Research Unit, South African Medical Research Council, Pretoria, South Africa

${ }^{2}$ Department of Geography, Geoinformatics \& Meteorology, University of Pretoria, Pretoria, South Africa

*Author for correspondence: Tel.: +27 12339 8543; cwright@mrc.ac.za

\author{
"research, skills development and treatment, and education and raising awareness need to be \\ addressed if we are to reduce the health disparities that are seen in overlooked populations and \\ minority groups in relation to melanoma"
}

First draft submitted: 29 May 2018; Accepted for publication: 31 May 2018; Published online: 10 July 2018

Keywords: health disparities • melanoma • minority groups • pigmented skin • skin cancer

Over the past 25 years, we have made progress in melanoma treatment, screening, diagnosis and prevention, however, addressing melanoma health disparities in some population groups is an area in which progress has not kept up [1]. In general, poorer outcomes for melanoma exist for ethnic minorities, people who are less educated, people of lower socio-economic status, the elderly and the uninsured [2]. Ethnic minorities are thought to be about two- to three-times as likely to die from melanoma as age- and sex-matched individuals from nonminority groups [3]. Biological factors may account for some of these differences but several of the underlying mechanisms of these disparities are unclear and under-researched. Furthermore, while numerous epidemiological studies have been carried out on melanoma and its prevention [4,5] we lack comprehensive research on melanoma in ethnic minorities and other overlooked population groups in countries around the world [2].

There is a need to address these disparities in melanoma in overlooked population groups. In countries where there are typically few dermatologists serving the population, the existing dermatology workforce may face additional pressure if melanoma incidence increases and the patient load increases [1]. We may see more overlooked populations seeking assistance with screening and detection of melanoma. In some countries, changing demographics may also mean larger proportions of the population falling into ethnic minority groups, therefore numbers of people seeking healthcare visits may increase. In addition to these pressures, premature deaths [6], lost productivity due to years of life lost [7] and impacts on communities and families are also costs associated with melanoma disparities in overlooked groups.

In general, primary and secondary melanoma prevention has focused most of its attention on specific populations and at-risk groups based on the known nonmodifiable and modifiable risk factors for melanoma, among others, presence of naevi, sun exposure, sunbed use, sunburns and phenotype [8,9]. Population-based melanoma prevention messages alerting the public to these risk factors have been largely directed to the general population as well as to high-risk outdoor occupations, high-risk recreation groups, patients postskin cancer diagnosis and organ transplant recipients [10]. Overlooked groups include ethnic minorities, the elderly, the military, the uninsured and people with disabilities [11,12]. Among the elderly, the perceived risk for developing melanoma and inclination to seek a skin examination tends to be low [1]. Health insurance status also influences melanoma outcomes where people seeing their primary care physician in the private sector are more likely to use skin cancer prevention screening, for example, compared with patients using public healthcare [13].

Freeman and Chu's health disparities cancer model is based on the premise that social setting, incorporating poverty, culture and social justice, plays a part in disease outcome [14]. The model infers a relation between social

Future Medicine 
determinants of health and health inequities [15]. Many of the factors related to the successful implementation of cancer prevention, control, and treatment are affected by socially determined factors. One can consider povertyrelated barriers that influence melanoma outcomes such as income, education and health insurance, as well as barriers related to social injustice, for example, incorrect assumptions and mistrust [2]. Some barriers influencing melanoma outcomes pertain to culture including risk behaviors, acculturation, skin cancer awareness and knowledge, language fluency and perceptions of skin cancer risk [2]. In general, people with deeply pigmented skin usually perceive that skin cancer is a disease that only affects people with fair skin [16] and while this is in part true since melanoma incidence is higher in people with fair skin [17], melanoma does occur in people with deeply pigmented skin. However, the risk factors associated with melanoma in people with deeply pigmented skin are, in general, poorly understood.

\section{Exploring melanoma disparities in people with deeply pigmented skin: a South African perspective}

South Africa has a dual economy with both formal and informal sectors and is a country with one of the highest inequality rates in the world. In 2014, the Gini coefficient measuring relative wealth for South Africa was 0.69 ( 1 is perfectly unequal) based on income data including salaries, wages and social grants [18]. In addition to an unequal economy, the South African population of 55.7 million comprises four population groups: $80 \%$ Black African, 9\% colored (of mixed ancestry), 8\% White and 3\% Indian/Asian [19]. About 82 out of every 100 people of the country's population seek healthcare in the public sector and only 18 in 100 people have private medical insurance [20]. Furthermore, South Africa is said to battle a quadruple burden of disease that includes HIV/AIDS, high-material and child mortality, high levels of violence and injuries and a growing burden from noncommunicable diseases [21].

Finding studies and data on melanoma morbidity and mortality for overlooked groups in South Africans is challenging. National Cancer Registry data show that melanoma incidence is lower among Black Africans in South Africa compared with White South Africans [17]. The type of melanoma and the anatomic site of melanoma occurrence also differs between population groups, where this information is available. Melanoma in Black Africans is usually acral lentigenous melanoma and commonly occurs on the upper limb/shoulder and lower limb/hip compared with the occurrence on the head and trunk anatomic sites of White South Africans [17]. The 5-year melanoma survival for people with deeply pigmented skin is typically lower compared with that for White populations [2]. The few published studies show later stage of cutaneous melanoma at diagnosis and lower 5-year survival rates among Black South Africans compared with White South Africans [22]. The risk factors for melanoma in people with dark skin are complex and not well understood. It is unlikely that sun exposure plays an important role in the epidemiology of acral lentigenous melanoma, however, trauma, such as insect bites, wounds and scar tissue, may be an important risk factor warranting further research for this population group [22]. Other risk factors such as sex, age and genetics are also important [22].

Contributing factors related to delayed diagnosis seen among Black South Africans are likely to include lowperceived melanoma risk, low index of suspicion of melanoma among healthcare providers and in some cases, suboptimal access to healthcare [22]. If efforts were made to increase secondary melanoma prevention in South Africa and improve early detection of thin melanomas, there may be some pressure placed on the 180 dermatologists practicing in the public and private sectors in the country [23]. If we are to try and address melanoma in Black South Africans, a comprehensive, holistic approach will be needed.

\section{What can be done to reduce health disparities in melanoma in overlooked populations?}

The complexities of preventing melanoma in overlooked population groups, such as Black South Africans and in countries with wide disparities in inequality are challenging [24]. Three board areas, namely research, skills development and treatment, and education and raising awareness need to be addressed if we are to reduce the health disparities that are seen in overlooked populations and minority groups in relation to melanoma. Reliable data and research are needed on the epidemiology of melanoma in overlooked population groups in different countries around the world. For example, we need to explore how socially determined factors influence the inequities that exist in stage of melanoma and survival rates among individuals with dark versus fair skin. There is work to be done to better understand emerging issues, such as the implications of skin bleaching among individuals with dark skin and the subsequent risk, if any, of skin cancer [25]. It would also be helpful if cancer registry records include 
data about personal ancestry, population group and skin color. This information would be valuable for developing effective interventions and developing melanoma prevention measures and strategies that target vulnerable groups.

We need to improve knowledge and awareness of melanoma among medical professionals [12] as well as familiarize clinicians with important features that characterize melanoma and the different forms of melanoma in patients across all Fitzpatrick phototypes with different skin colors [12]. It is also important that we aim to raise awareness about dermatologic health disparities in melanoma among overlooked populations themselves and ensure high-quality treatment and healthcare services for all groups.

Many primary and secondary population-based melanoma prevention campaigns have focused on targeting the general population and provided specific messages, such as to check moles, to avoid sunburn and to use sun protection. Messages about sunburn, for example, might not be relevant in terms of melanoma prevention among some overlooked groups, especially for individuals with deeply pigmented skin. Sunburn as a term is not commonly understood among individuals with deeply pigmented skin [26] therefore, messages about sun protection related to sunburn are not helpful to all individuals in a population in relation to melanoma prevention. We need to enhance and expand our efforts to raise awareness about melanoma among ethnic minorities and overlooked groups and include information on early signs of melanoma in pigmented skin [12]. This information needs to be in appropriate formats for different cultures and languages too [16].

\section{Conclusion}

The complexities of preventing skin cancers in countries with multiethnic populations and wide disparities in inequality, wealth, health status and access to health services are challenging. Several factors contribute to these disparities in melanoma outcomes including social determinants of health and various barriers such as the lack of training for diagnosis and treatment of melanoma among healthcare professionals in public healthcare settings. Next generation melanoma prevention research should include, among others, efforts to identify risk factors for melanoma in overlooked population and populations with disparities in melanoma outcomes. Several of the barriers, while undoubtedly complex, are potentially modifiable and additional research will help to better understand how to tackle these complexities with the end goal of reducing the burden of melanoma morbidity and mortality in overlooked populations and ethnic minorities.

\section{Acknowledgements}

This commentary was developed from a plenary presentation given at the 4th International UV and Skin Cancer Prevention conference held at Ryerson University in Toronto, Ontario, Canada from the 1-4 May 2018.

\section{Financial \& competing interests disclosure}

CY Wright receives research funding support from the South African Medical Research Council and the National Research Foundation of South Africa. The author has no other relevant affiliations or financial involvement with any organization or entity with a financial interest in or financial conflict with the subject matter or materials discussed in the manuscript apart from those disclosed.

No writing assistance was utilized in the production of this manuscript.

\section{Conflict of interest}

The author declares that there are no conflicts of interest.

\section{Open access}

This work is licensed under the Attribution-Noncommercial-Noderivatives 4.0 Unported license. To view a copy of this license, visit http://creativecommons.org/licenses/by-nc-nd/4.0/

\section{References}

Papers of special note have been highlighted as: $\bullet$ of interest; $\bullet \bullet$ of considerable interest

1. Buster KJ, Stevens EI, Elmets CA. Dermatologic health disparities. Dermatol. Clin. 30, 53-58 (2012).

2. Harvey VM, Patel, H Sandhu S et al. Social determinants of racial and ethnic disparities in cutaneous melanoma outcomes. Cancer Control 21, 343-349 (2014).

-. Provides in-depth detail of the social determinants of and disparities in melanoma outcomes and gives excellent examples.

3. Kundu RV, Kamaria M, Ortiz S, West DP, Rademaker AW, Robinson JK. Effectiveness of a knowledge-based intervention for melanoma among those with ethnic skin. J. Am. Acad. Dermatol. 62(5), 777-784 (2010). 
4. Berwick M, Buller DB, Cust A et al. Melanoma epidemiology and prevention. Melanoma 167, 17-49 (2015).

5. Rastrelli M, Tropea S, Rossi CR, Alaibac M. Melanoma: epidemiology, factors, pathogenesis, diagnoses and classification. In vivo 28, 1005-1011 (2014)

6. Ekwueme DU, Guy GP, Lu C, Rim SH, Parelkar P, Chen SC. The health burden and economic costs of cutaneous melanoma mortality by race/ethnicity-United States, 2000-2006. J. Am. Acad. Dermatol. 65, S133.e1-S133.e12 (2011).

7. Hanly PA, Sharp L. The cost of lost productivity due to premature cancer-related mortality: an economic measure of the cancer burden. BMC Cancer 14, 224 (2014).

8. Gandini S, Sera F, Cattaruzza MS et al. Meta-analysis of risk factors for cutaneous melanoma: III. Family history, actinic damage and phenotypic factors. Eur. J. Cancer. 41, 2040-2059 (2005).

9. Gandini S, Sera F, Cattaruzza MS et al. Meta-analysis of risk factors for cutaneous melanoma: II. Sun exposure. Eur. J. Cancer. 41, 45-60 (2005).

10. Chung CL, Nadhan KS, Shaver CM et al. Comparison of post-transplant dermatologic disease by race. JAMA Dermatol. 153, 552-558 (2017).

11. Riemenschneider K, Liu J, Powers JG. Skin cancer in the military: a systematic review of melanoma and nonmelanoma skin cancer incidence, prevention, and screening among active duty and veteran personnel. J. Am. Acad. Dermatol. 78(6), 1185-1192 (2018).

12. Hu S. Are we overlooking skin cancer in ethnic minorities? Cutaneous Oncol. Today 11, 5-8 (2011).

-. Provides insight into skin cancer disparities in ethnic minorities and suggestions with which to address inequalities.

13. Roetzheim RG, Pal N, Tennant C et al. Effects of health insurance and race on early detection of cancer. J. Natl Cancer Inst. 91(17), 1409-1415 (1999).

14. Freeman HP, Chu KC. Determinants of cancer disparities: barriers to cancer screening, diagnosis, and treatment. Surg. Oncol. Clin. N. Am. 14, 655-669 (2002).

15. Marmot M. Social determinants of health inequities. Lancet 9464, 1099-1104 (2005).

16. Ndlova NC, Gathers R, Tsoka-Gwegweni J, Hift RJ. Skin cancer awareness and sunscreen use among outpatients of a South African hospital: need for vigorous public education. S. Afr. Fam. Prac. 1(1), 1-5 (2018).

17. Norval M, Kellet P, Wright CY. The incidence and body site of skin cancers in the population groups of South Africa. Photoderm. Photoimmunol. Photomed. 30(5), 262-265 (2014).

18. World Bank. South Africa overview. (2018). www.worldbank.org/en/country/southaf rica/overview

19. Statistics South Africa. Mid-year population estimates 2017. (2018). www.statssa.gov.za/publications/P0302/P03022017.pdf

20. Statistics South Africa. General household survey 2016. (2018). www.statssa.gov.za/?p=10548

21. Mayosi BM, Flisher AJ, Lalloo UG, Sitas F, Tollman SM, Bradshaw D. The burden of non-communicable diseases in South Africa. Lancet 374, 934-947 (2009).

22. Norval M, Wright CY. The epidemiology of cutaneous melanoma in the white and black african population groups in South Africa. In: Cutaneous Melanoma: Etiology and Therapy. Ward and Farma (Eds). Condon Publishers, Brisbane,Australia (2017).

23. Econex. Updated GP and Specialist Numbers: 2011 and 2012. (2018). www.hpcsa.co.za/Uploads/editor/UserFiles/downloads/service_f ees-tariff/submissions/sappf_f_econex_updated_gp_specialist_numbers_27\%2003\%202013.pdf

24. Benatar S. The challenges of health disparities in South Africa. S. Afr. Med. J. 103(3), 154-155 (2013).

- Discusses health disparities in a wider context beyond those related to melanoma outcomes.

25. Benn EKT, Alexis A, Mohamed N, Wang Y-H, Khan IA, Liu B. Skin bleaching and dermatologic health of African and Afro-Caribbean populations in the US: new directions for methodologically rigorous, multidisciplinary and culturally sensitive research. Dermatol. Ther. (Heidelb) 6(4), 453-459 (2016).

26. Wilkes M, Wright CY, du Plessis JL, Reeder AI. Fitzpatrick skin type, individual typology angle and melanin index in an African population: taking steps toward universally applicable skin photosensitivity assessments. JAMA Dermatol. 151(8), 902-903 (2015). 